\title{
A COOPERATIVE SENSOR NETWORK: OPTIMAL DEPLOYMENT AND FUNCTIONING
}

\author{
Alfonso Farina $^{1}$, Antonio Graziano ${ }^{2}$, \\ FrANCESCA MARIANI $^{3 *}$ AND FranCESCO Zirilli ${ }^{4}$ \\ Communicated by A. Farina
}

\begin{abstract}
A network of mobile cooperative sensors is considered. The following problems are studied: (1) the "optimal" deployment of the sensors on a given territory; (2) the detection of local anomalies in the noisy data measured by the sensors. In absence of an information fusion center in the network, from "local" interactions between sensors "global" solutions of these problems are found.
\end{abstract}

Keywords. Sensors network, Voronoi partition, statistical test.

Mathematics Subject Classification. 90C30, 90C60, 62F03.

\section{INTRODUCTION}

Sensor networks have countless applications, for example, we mention the sensor networks used in computer science and telecommunications, in biology, where they can be used to monitor the behaviour of animal species such as birds or fishes, and in habitat monitoring, where they can be used to provide real-time rainfall

Received February 19, 2010. Accepted November 10, 2010.

1 SELEX Sistemi Integrati s.p.a., via Tiburtina km 12 400, 00131 Roma, Italy.

afarina@selex-si.com

2 SELEX Sistemi Integrati s.p.a., via Tiburtina km 12 400, 00131 Roma, Italy.

agraziano@selex-si.com

3 CERI - Centro di Ricerca "Previsione Prevenzione e Controllo dei Rischi Geologici", Università di Roma "La Sapienza", Piazza Umberto Pilozzi 9, 00038 Valmontone (RM), Italy. fra_mariani@libero.it

4 CERI - Centro di Ricerca "Previsione Prevenzione e Controllo dei Rischi Geologici", Università di Roma "La Sapienza", Piazza Umberto Pilozzi 9, 00038 Valmontone (RM), Italy. f.zirilli@caspur.it

* The work of this author has been partially supported by SELEX Sistemi Integrati s.p.a.

(Roma, Italy) through a research contract granted to CERI-Università di Roma "La Sapienza". 
and water level information used to evaluate the possibility of flooding. To fix the ideas as an example of sensor network we consider a collection of devices (i.e.: sensors) placed on a given geographical area, that will be called territory, to monitor environmental conditions, such as temperature, pressure, sound at different positions. We consider mobile cooperative sensor networks, that is networks made of sensors able to move on the territory to achieve a desired optimal deployment configuration and willing to cooperate to pursue a goal. The goal pursued is the construction of "consensus" values of the quantities monitored and of the magnitude of the noise contained in the data. These consensus values are used to detect local anomalies in the noisy data measured. Each sensor is imagined as being placed on a node of a graph and communicates with the sensors located in the nodes of the graph adjacent to the node where it is. The problem of determining the representation and the topology of the graph associated to the network, that is the problem of determining the spatial coordinates of the nodes of the graph where the sensors are located and the set of the branches of the graph, is called deployment problem. The quality of the territory monitoring obtained with the sensor network depends on the way the sensors are deployed. In many circumstances it is important to guarantee an high quality monitoring. We assume that high quality monitoring means that the sensors are "well distributed" on the territory monitored and that the communications on the network are not "too heavy". The deployment problem will be formulated as an optimization problem. The deployment resulting as solution of this optimization problem will be called optimal deployment. This optimal deployment guarantees an high quality territory monitoring. After being deployed on the territory the sensors start monitoring noisy data. We assume the sensors to be identical. Just to fix the ideas let us imagine that the sensors measure the temperature of the territory in the position or in the "area" where they are located and that in absence of anomalies there is a uniform temperature on the territory where the sensors are deployed. The sensor measures are synchronous and noisy. This measurement process is repeated periodically in time with a given frequency. From these measures we want to deduce a kind of "consensus" temperature of the territory where the sensors are deployed and an estimate of the magnitude of the noise contained in the data. Finally using these consensus values as reference values we want to detect local anomalies in the data measured by the individual sensors. In the detection of these anomalies we will make some elementary hypotheses in order to be able to use elementary statistical tests. Through these statistical tests a (statistical) significance will be associated to the anomalies detected.

Usually in sensor networks the information traffic goes from the sensor nodes to a single sink node called information fusion center. According to the information received from the sensors the fusion center monitors the area where the sensors are deployed and decides the actions to take. In this type of networks the fusion center will be responsible for the deployment of the sensors on the territory and for the elaboration of the noisy data measured. If an intruder reaches and neutralizes the fusion center, the communication between the network nodes are interrupted and the whole network is exposed to the risk of becoming useless as a network 
even if the individual sensors may still be all working. In many circumstances the problem of detecting the presence of intruders in a monitored territory is very important. In this paper the intruder detection problem will be identified with the problem of the detection of local anomalies in the noisy data measured by the sensors. That is we assume that the local anomalies detected in the data are due to the presence of intruders.

In recent years the decreasing sensor cost and the development of telecommunication technologies have made possible the deployment of networks with a huge number of sensors. The use of information fusion centers is unpractical when networks made of a huge number of sensors are considered. These facts have determined the emergence of a new class of sensor networks whose way of functioning is called "network centric". These networks do not have a fusion center and their functioning is based on the information exchange between "near by" sensors. In a well designed network centric network global solutions to the problems considered are obtained from local interactions. Note that a sensor network operated in a network centric manner can tolerate the lost of some of its sensors without loosing its character of being a network, that is without loosing its functionality.

In this paper we present a mathematical model for the deployment of a sensor network, for the creation of consensus values from the noisy data measured and a statistical methodology to detect local anomalies in these data. Remind that we have assumed that a local anomaly in the data is associated to the presence of an intruder. In future works also the problem of taking an action after having detected an intruder will be considered. The action taken will consist in moving some (mobile) sensors to meet the intruder, this last problem is called rendez vous problem. The model of sensor network presented in this paper is characterized by the absence of a fusion center. In other words the deployment found, the construction of the consensus values and the detection of local anomalies in the data are the result of local interactions between sensors. Nevertheless the local interactions will lead to global solutions of the problems considered. That is our model is a model of a network centric sensor network.

In Section 2 we study the deployment problem. In Section 3 we study the problem of the creation of the consensus values and of the detection of local anomalies in the data measured.

\section{The DEPLOYMENT PROBLEM}

Let $\Omega$ be a bounded connected polygonal domain contained in the two dimensional real Euclidean space $\mathbb{R}^{2}$. The domain $\Omega$ represents the territory where the sensor network must be deployed. Let $\|\cdot\|$ be the Euclidean norm of · in $\mathbb{R}^{2}$.

We consider $N$ sensors $s_{1}, s_{2}, \ldots, s_{N}$, located, respectively, in the points $\underline{\xi}_{1}, \underline{\xi}_{2}, \ldots$, $\underline{\xi}_{N} \in \Omega$. The points $\underline{\xi}_{1}, \underline{\xi}_{2}, \ldots, \underline{\xi}_{N} \in \Omega$ are assumed to be distinct. To the sensor network deployed in the points $\underline{\xi}_{1}, \underline{\xi}_{2}, \ldots, \underline{\xi}_{N}$ corresponds a graph whose nodes are the sensor locations and whose branches join the sensors able to communicate between themself. This graph is assumed to be connected and can be imagined as 


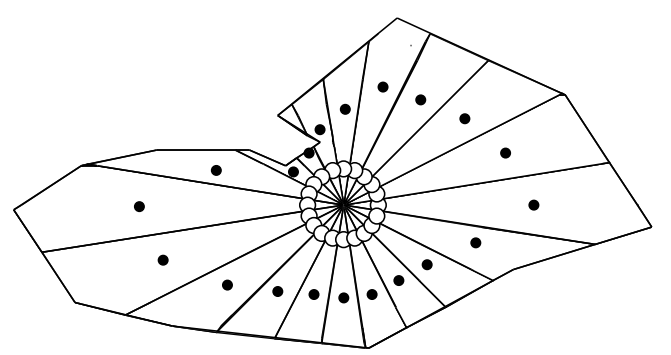

(a)

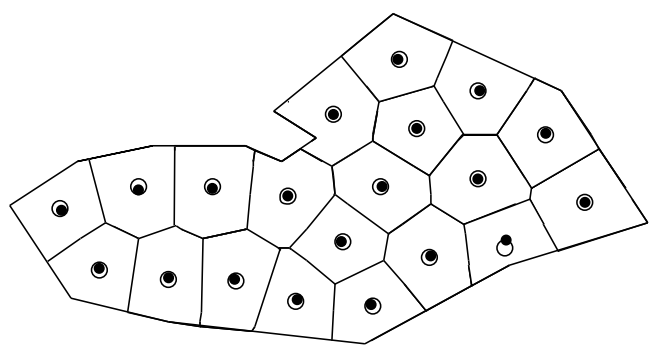

(b)

FiguRE 1. (a) Voronoi partition of $\boldsymbol{\Omega}$ and (b) optimal Voronoi partition of $\Omega$.

laying on the territory. The assumption that the graph is connected is equivalent to assuming that the sensors constitute a network. For $i=1,2, \ldots, N$ we associate to the sensor $s_{i}$ a polygonal region $\Omega_{i} \subset \Omega$ defined by the condition that the points belonging to $\Omega_{i}$ are closest to the sensor $s_{i}$, that is they are closest to $\underline{\xi}_{i}$, than to any other of the remaining sensors $s_{j}$ located in $\underline{\xi}_{j}, j \neq i, j=1,2, \ldots, \hat{N}$. That is we have:

$$
\begin{aligned}
\Omega_{i}= & \left\{\underline{x} \in \Omega: i=\operatorname{argmin}\left(f(j)=\left\|\underline{x}-\underline{\xi}_{j}\right\|\right)\right. \\
& j=1,2, \ldots, N\}, \quad i=1,2, \ldots, N
\end{aligned}
$$

When for a given $\underline{x} \in \Omega$ the minimizer of the function $f(j)=\left\|\underline{x}-\underline{\xi}_{j}\right\|, j=$ $1,2, \ldots, N$, is not unique we attribute $\underline{x}$ to $\Omega_{i}$, where $i$ is the smallest index between the indices that are minimizers of the function $f$.

The collection of subsets $\left\{\Omega_{1}, \Omega_{2}, \ldots, \Omega_{N}\right\}$ defined in (2.1) and further specified by the condition above is a partition of $\Omega$ and is called Voronoi partition of $\Omega$ associated to the Voronoi centers $\underline{\xi}_{1}, \underline{\xi}_{2}, \ldots, \underline{\xi}_{N}$ (see Fig. 1) [1]. The sets $\Omega_{1}, \Omega_{2}, \ldots, \Omega_{N}$ are called Voronoi cells. Remind that $\underline{\xi}_{i} \in \Omega_{i}, i=1,2, \ldots, N$. The sensor $s_{i}$ is located in $\underline{\xi}_{i}$ and monitors the subregion $\Omega_{i}$ of $\Omega, i=1,2, \ldots, N$. Note that there is a Voronoi partition of $\Omega$ associated to each choice of the Voronoi centers $\underline{\xi}_{1}, \underline{\xi}_{2}, \ldots, \underline{\xi}_{N}$. 
We can conclude that each choice of the Voronoi centers $\underline{\xi}_{1}, \underline{\xi}_{2}, \ldots, \underline{\xi}_{N} \in \Omega$ completed with the graph that defines the communication between the sensors is a deployment of the sensors $s_{1}, s_{2}, \ldots, s_{N}$ on the territory $\Omega$.

We want to determine a Voronoi partition of $\Omega$ that is optimal with respect to a pre-specified criterion. The criterion considered is the fact that the Voronoi centers $\underline{\xi}_{1}, \underline{\xi}_{2}, \ldots, \underline{\xi}_{N}$ should coincide (as much as possible) with the centers of mass of the corresponding Voronoi cells $\Omega_{1}, \Omega_{2}, \ldots, \Omega_{N}$. This property translates in mathematical terms the request that the sensors are well distributed on the territory. That is we call optimal Voronoi partition the Voronoi partition associated to the Voronoi centers whose coordinates $\underline{\xi}_{1}^{*}, \underline{\xi}_{2}^{*}, \ldots, \underline{\xi}_{N}^{*}$ are the solution of the following problem:

$$
\min _{\underline{\xi}_{1}, \underline{\xi}_{2}, \ldots, \underline{\xi}_{N}} F\left(\underline{\xi}_{1}, \underline{\xi}_{2}, \ldots, \underline{\xi}_{N}\right)=\sum_{j=1}^{N}\left\|\underline{B}_{j}-\underline{\xi}_{j}\right\|^{2}
$$

subject to the constraints:

$$
\underline{\xi}_{i} \neq \underline{\xi}_{j}, \quad i \neq j \quad i, j=1,2, \ldots, N
$$

where $\underline{B}_{j}$ is the center of mass of the Voronoi cell $\Omega_{j}, j=1,2, \ldots, N$. Moreover we require:

$$
F\left(\underline{\xi}_{1}^{*}, \underline{\xi}_{2}^{*}, \ldots, \underline{\xi}_{N}^{*}\right)=0
$$

that is, we require that the Voronoi centers and the centers of mass of the Voronoi cells coincide. Note that in general $\underline{B}_{j}$ depends on $\underline{\xi}_{1}, \underline{\xi}_{2}, \ldots, \underline{\xi}_{N}$ and that the function $F\left(\underline{\xi}_{1}, \underline{\xi}_{2}, \ldots, \underline{\xi}_{N}\right)$ is in general a nonlinear function of $\underline{\xi}_{1}, \underline{\xi}_{2}, \ldots, \underline{\xi}_{N}$. The solution of problem (2.2), (2.3), (2.4) after having specified the communications between the sensors is the optimal deployment announced in the Introduction (see Figs. $1 \mathrm{~b}$ and 2). When we are unable to satisfy condition (2.4) we accept the available solution of (2.2), (2.3) as location of the Voronoi centers corresponding to the optimal deployment. Note that in general the solution of problem (2.2), (2.3), (2.4) is non unique.

In order to solve the optimization problem (2.2), (2.3), (2.4) we use the continuous analog of the steepest descent method [5]. The steepest descent method when used to solve problem (2.2) is an iterative procedure that, beginning from an initial guess, updates at every iteration the current approximation of the solution of (2.2) with a step in the direction of the gradient of the function $F\left(\underline{\xi}_{1}, \underline{\xi}_{2}, \ldots, \underline{\xi}_{N}\right)$. In the deployment problem considered the steepest descent method must be adapted to the presence of the constraints (2.3), of the condition (2.4) and to the requirement that its implementation must lead to a network centric solution of the deployment problem. In this paper to keep the exposition simple we will not discuss how to impose (2.3). A treatment of constraints in the continuous analog of the steepest descent algorithm can be found in [4]. Note also that the solutions of (2.2), (2.3) that are of interest are usually interior points of the constraints (2.3). That is the constraint issue usually is not relevant in the solution of (2.2), (2.3). Similarly we will not pay attention to condition (2.4). In fact with respect to (2.4) we will simply verify if the solution of the optimization problem determined by the steepest 


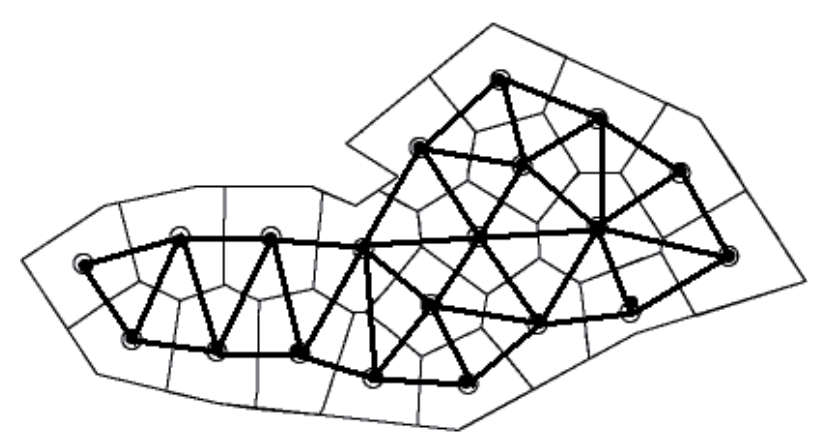

Figure 2. Graph associated to the optimal Voronoi partition of $\boldsymbol{\Omega}$ shown in Figure 1b.

descent method satisfies (2.4). Let us concentrate our attention on the issue of building a network centric implementation of the continuous analog of the steepest descent method to solve (2.2).

We assume that the sensor $s_{i}$ knows only the position of its neighbour sensors, that is of the sensors that belong to a disk with center $\underline{\xi}_{i}$ and radius $r>0$, $i=1,2, \ldots, N$. Later we will show how to choose $r$. The solution of the optimization problem (2.2) is found approximating the solution of the system of differential equations:

$$
\underline{\xi}_{i}=-\nabla_{\underline{\xi}_{i}} F\left(\underline{\xi}_{1}\left(\lambda_{1}\right), \underline{\xi}_{2}\left(\lambda_{2}\right), \ldots, \underline{\xi}_{N}\left(\lambda_{N}\right)\right), \lambda_{1}>0, i=1,2, \ldots, N,
$$

where $\lambda_{1}$ denotes a real parameter, with the solution of the "network centric" system of differential equations:

$$
\underline{\xi}_{i}=-\nabla_{\underline{\xi}_{i}} F_{i}\left(\underline{\xi}_{1}\left(\lambda_{1}\right), \underline{\xi}_{2}\left(\lambda_{2}\right), \ldots, \underline{\xi}_{N}\left(\lambda_{N}\right)\right), \lambda_{1}>0, i=1,2, \ldots, N,
$$

with

where

$$
F_{i}=\sum_{j \in L_{i}}\left\|\underline{\tilde{B}}_{i, j}-\underline{\xi}_{j}\right\|^{2}, \quad i=1,2, \ldots, N
$$

$$
\begin{gathered}
L_{i}=\left\{\underline{\xi}_{j}, j=1,2, \ldots, N: \underline{\xi}_{j} \text { is neighbour to } \underline{\xi}_{i}\right\}, \\
i=1,2, \ldots, N,
\end{gathered}
$$

and $\underline{\tilde{B}}_{i, j}$ is the center of mass of the Voronoi cell $\tilde{\Omega}_{i, j}$ obtained computing the Voronoi partition of $\Omega$ associated to the Voronoi centers $\underline{\xi}_{j}, j \in L_{i}, i=1,2, \ldots, N$. Let us assume that $r>0$ is large enough to guarantee that $\underline{\xi}_{j}$ is neighbour of $\underline{\xi}_{i}$ when the distance between $\Omega_{j}$ and $\Omega_{i}$ is zero, $i, j=1,2, \ldots, N$. Note that with this assumption we have $\underline{\tilde{B}}_{i, i}=\underline{B}_{i}, i=1,2, \ldots, N$. In (2.5), (2.6) the ' denotes differentiation with respect to $\lambda_{1}$. We observe that (2.5) is known as the steepest descent 
differential equation and the continuous analog of the steepest descent method consists in obtaining the solution of the optimization problem (2.2) computing the asymptotic value as $\lambda_{1}$ goes to infinity of a solution of (2.5) equipped when $\lambda_{1}=0$ with a suitable initial condition. This asymptotic value hopefully is a point that solves (2.2) and satisfies (2.3) and (2.4).

Note that the function $F_{i}$ depends only on $\underline{\xi}_{j}, j \in L_{i}$, that is can be computed in the location $\underline{\xi}_{i}$ using only information available in $\underline{\xi}_{i}, i=1,2, \ldots, N$. Approximating the gradient of $F$ with the appropriate pieces of the gradients of the functions $F_{i}$, $i=1,2, \ldots, N$, and using (2.6) instead than (2.5) we can find an approximation of the solution of (2.2) integrating numerically the initial value problem for (2.6). Note that the solution of the $i$ th differential equation of (2.6) is computed in the location $\underline{\xi}_{i}, i=1,2, \ldots, N$. This approximation of the solution of (2.2) is obtained using only local information so that it is "network centric". When the asymptotic value as $\lambda_{1}$ goes to infinity of the solution of (2.6) considered coincides with an asymptotic value of a solution of (2.5), solving numerically (2.6), we can obtain in a network centric manner a solution of (2.2).

The choices of the optimal Voronoi partition and of the steepest descent method to determine it are only one of the many other legitimate choices. A comparison between some of these choices will be presented elsewhere.

Note that we have introduced a kind of "network centric" steepest descent method to solve the optimization problem (2.2), (2.3) that models the deployment problem. Similarly other well known methods of nonlinear optimization such as the Newton method or the quasi Newton methods could be transformed into "network centric" methods and used to solve the deployment problem. Moreover when we add condition (2.4) to problem (2.2), (2.3) the problem becomes a global optimization problem while the methods mentioned above are only local methods. That is: there is room for the development of network centric global optimization methods. However, for the purpose of the elementary application discussed below and shown in Figure 1, the "network centric" steepest descent method seems to be good enough. Note that several simplifying assumptions are used in the deployment problem considered above, for example: the region $\Omega$ is homogeneous, there are no constraints on the movement of the sensors in $\Omega$, the only constraint to the positioning of the sensors in $\Omega$ is expressed by (2.3). In many practical circumstances these assumptions are unrealistic. It is easy to see how to reformulate the optimization problem (2.2), (2.3) and the condition (2.4)to model more realistic situations.

In Figure 1 the polygonal region shown represents $\Omega$, we choose $N=20$ and for $i=1,2, \ldots, 20$ we denote with the full circle the position of the center of mass $\underline{B}_{i}$ of the subset $\Omega_{i}$ (shown in the figure), and with the empty circle the position of the sensor $\underline{\xi}_{i}$. In Figure 1a we show the Voronoi partition of the the domain $\Omega$ associated to 20 Voronoi centers $\underline{\xi}_{1}, \underline{\xi}_{2}, \ldots, \underline{\xi}_{20}$ shown and the corresponding centers of mass $\underline{B}_{1}, \underline{B}_{2}, \ldots, \underline{B}_{20}$ of the associated Voronoi cells $\Omega_{1}, \Omega_{2}, \ldots, \Omega_{20}$. Note that in Figure 1a we have $\underline{\xi}_{i} \neq \underline{B}_{i}, i=1,2, \ldots, 20$. In Figure $1 \mathrm{~b}$ we show an optimal Voronoi partition. Note that in Figure $1 \mathrm{~b}$ we have $\underline{\xi}_{i}=\underline{B}_{i}, i=1,2, \ldots, 20$. The Voronoi 
partition shown in Figure 1b satisfies (2.2), (2.3), (2.4). The centers of Figure 1b have been obtained integrating numerically using the explicit Euler method (2.6) equipped with the initial condition given by the centers shown in Figure 1a. In Figure 2 we show the graph associated to the optimal Voronoi partition of $\Omega$ shown in Figure 1b. The graph is obtained joining with branches the Voronoi centers that are (distinct) neighbours. In Figures 1 and 2 we have chosen $r=k(\operatorname{area}(\Omega))^{1 / 2}$, where $k$ is a parameter that can be changed during the optimization procedure used to solve (2.2), (2.3), (2.4). The way of choosing $k$ will not be discussed here.

\section{The Consensus values And the Detection}

Remind that we have assumed that the graph $G$ associated to the optimal deployment determined in Section 2 is connected (see Figs. 2 and 1b). Moreover we remind that, since there is not a fusion center, each node of the graph $G$ does not know the positions of all the remaining nodes of the graph, in fact it knows only the positions of its neighbour nodes. Let $L$ be the Laplacian matrix associated to $G$ [2]. The matrix $L$ is a symmetric positive semidefinite $N \times N$ matrix. Let $\underline{x}\left(\lambda_{2}\right)=\left(x_{1}\left(\lambda_{2}\right), x_{2}\left(\lambda_{2}\right), \ldots, x_{N}\left(\lambda_{2}\right)\right)^{\mathrm{T}}, \lambda_{2}>0$, be a real $N$ dimensional vector depending on the real parameter $\lambda_{2}$. The superscript ${ }^{\mathrm{T}}$ means transposed. We consider the system of ordinary differential equations:

$$
\underline{\dot{x}}\left(\lambda_{2}\right)=-L \underline{x}\left(\lambda_{2}\right), \quad \lambda_{2}>0,
$$

equipped with the initial condition:

$$
\underline{x}(0)=\underline{\alpha},
$$

where $L \underline{x}$ denotes the usual matrix vector multiplication, $\underline{\alpha}=\left(\alpha_{1}, \alpha_{2}, \ldots, \alpha_{N}\right)^{\mathrm{T}}$ is a known initial condition and denotes differentiation with respect to $\lambda_{2}$. Since $G$ is connected we have:

$$
\lim _{\lambda_{2} \rightarrow+\infty} x_{i}\left(\lambda_{2}\right)=\frac{1}{N} \sum_{j=1}^{N} \alpha_{j}, \quad i=1,2, \ldots, N,
$$

where $\underline{x}\left(\lambda_{2}\right), \lambda_{2}>0$, is the solution of (3.1), (3.2). This result follows easily from the spectral properties of $L$ [2]. Note that the right hand side of (3.3) is the "average" of the initial condition $\underline{\alpha}$. Note that (3.1) can be interpreted as the "heat equation" on the graph $G$, that the problem (3.1), (3.2) can be seen as an initial value problem for the heat equation on $G$ and that (3.3) can be understood as the approach to an asymptotic equilibrium "temperature" in an "heat transfer" problem.

We assume that during the monitoring phase the sensor $s_{i}$ measures a physical quantity, such as, for example, the temperature, of the region $\Omega_{i}$ where it is located, $i=1,2, \ldots, N$. The sensors are identical, the measures made by the sensors are synchronous, repeated periodically in time and of course they are noisy. Moreover 
they are assumed to be independent. A first set of measures is taken by the sensors at time $t=t_{0}$ and is collected in the vector $\underline{\beta}_{0}=\left(\beta_{0,1}, \beta_{0,2}, \ldots, \beta_{0, N}\right)^{\mathrm{T}}$, where $\beta_{0, i}$ is the measure done by the sensor $s_{i}, i=1,2, \ldots, N$. The set of measures $\underline{\beta}_{0}$ will be used to obtain the "consensus" value $\bar{\beta}_{0}$ of the quantity monitored in $\bar{\Omega}$ at time $t=t_{0}$. We choose:

$$
\bar{\beta}_{0}=\frac{1}{N} \sum_{i=1}^{N} \beta_{0, i}
$$

Remind that the sensor $s_{i}$ located in $\underline{\xi}_{i}$ knows $\beta_{0, i}$ and communicates with the sensors $s_{j}$ located in $\underline{\xi}_{j}, j \in L_{i}, i=1,2, \ldots, N$. In order to provide to the sensor $s_{i}, i=1,2, \ldots, N$, the consensus value $\bar{\beta}_{0}$ in a network centric manner we proceed as follow: we choose $\underline{\alpha}=\underline{\beta}_{0}$ in (3.2) and we integrate numerically the initial value problem (3.1), (3.2) using the explicit Euler method in order to obtain a numerical approximation of $\lim _{\lambda_{2} \rightarrow+\infty} \underline{x}\left(\lambda_{2}\right)$. Note that the $i$ th differential equation of (3.1) is integrated in the location $\underline{\xi}_{i}$, and that using the explicit Euler method this can be done using only information available in the location $\underline{\xi}_{i}, i=1,2, \ldots, N$. Note that the analytic solution of (3.1), (3.2) is not "network centric" but its approximation with the explicit Euler method it is "network centric". Once obtained $\bar{\beta}_{0}$ we consider the vector $\underline{\gamma}_{0}=\frac{N}{N-1}\left(\left(\beta_{0,1}-\bar{\beta}_{0}\right)^{2},\left(\beta_{0,2}-\bar{\beta}_{0}\right)^{2}, \ldots,\left(\beta_{0, N}-\bar{\beta}_{0}\right)^{2}\right)^{\mathrm{T}}$, we choose $\underline{\alpha}=\underline{\gamma}_{0}$ in (3.2) and we integrate (3.1), (3.2) with the explicit Euler method as done above. In this way we obtain asymptotically a numerical approximation of $\bar{\gamma}_{0}$ where:

$$
\bar{\gamma}_{0}=\frac{N}{N-1} \sum_{i=1}^{N}\left(\beta_{0, i}-\bar{\beta}_{0}\right)^{2} .
$$

This approximation of $\bar{\gamma}_{0}$ is provided to each sensor in a network centric manner. Note that $\bar{\gamma}_{0}$ is an estimate of the magnitude of the noise contained in the data, in fact $\bar{\gamma}_{0}$ is the "sample" variance of the measures $\beta_{0, i}, i=1,2, \ldots, N$, made by the sensors at time $t=t_{0}$. The approximations of $\bar{\beta}_{0}$ and $\bar{\gamma}_{0}$ obtained integrating numerically (3.1), (3.2) are the consensus values announced in the Introduction. These values are "global" values (that is they depend on all the measures made by the sensor network at time $t=t_{0}$ ) and have been provided to each sensor in a network centric manner (that is using only "local" interactions between sensors).

The sensor $s_{i}$ repeats periodically in time the measure of the quantity of interest and after a given time interval has as its disposal a set of measures that can be compared with the consensus values $\bar{\beta}_{0}$ and $\bar{\gamma}_{0}$ to detect (local) anomalies, $i=$ $1,2, \ldots, N$. Let us assume that the set of measures made by the sensor $s_{i}$ is a sample taken from a set of independent identically distributed Gaussian random variables of mean $\mu_{i}$ and variance $\sigma_{i}^{2}, i=1,2, \ldots, N$. In these hypotheses the Student t-test and the Chi-square test [3] are the elementary statistical tools that must be used to compare $\mu_{i}$ and $\sigma_{i}^{2}$ (that are unknown) to $\bar{\beta}_{0}$ and $\bar{\gamma}_{0}, i=1,2, \ldots, N$. The result of this comparison is the detection of local anomalies. To the anomalies detected a (statistical) significance is associated. The statistical tests used are based on the assumption that the measures come from a set of independent identically 
distributed Gaussian random variables. Note that the estimators $\bar{\beta}_{0}$ and $\bar{\gamma}_{0}$ can be used in more general circumstances.

When we consider spatially distributed measurements made by sensor networks the hypothesis of that the sets of measurements studied are sampled from independent identically distributed Gaussian random variables is not satisfied in most of the practical cases. This hypothesis is made with the only purpose of making possible the use of elementary statistical tests (Student $\mathrm{T}$ and $\chi^{2}$ tests) in order to associate a statistical significance to the conclusions drawn from the measurements. When more realistic hypotheses are made about the statistical properties of the sets of measurements studied ad hoc statistical tests must be developed. These tests may involve the numerical solution of nontrivial optimization problems. The formulation of realistic hypotheses on the measurements and the development of the relevant ad hoc statistical tests is beyond the scope of this paper and will not be considered.

We conclude this paper noting that the work presented in this paper can be developed and extended in several directions. Let us mention some of them: (i) the study of the deployment problem in more general circumstances than those considered here; (ii) the strategy necessary to implement the action that must be taken when the presence of an intruder is detected; (iii) the study of the behaviour of networks with thousands or tens of thousands sensors.

For example in the case of a mobile intruder that pursues the goal of reaching a point in the territory $\Omega$ the attempt of some defenders (i.e.: sensors) of reaching the intruder is the so called rendez vous problem. This problem can be modeled as a differential game. The constraints of the game written as ordinary differential equations are simple forms of the Newton second law of dynamics. While some of the sensors are busy in the rendez vous attempt the remaining ones must be redeployed to keep control of the territory. The redeployment problem can be formulated as an optimization problem similar to problem (2.2), (2.3), (2.4) where some extra constraints are added to express the fact that some sensors are busy pursuing the rendez vous. Finally we point out that in order to make the model more realistic uncertainty can be introduced in the problems considered and in particular can be introduced in the differential game modeling the rendez vous.

\section{REFERENCES}

[1] F. Aurenhammer, Voronoi Diagrams - A Survey of a Fundamental Geometric Data Structure. ACM Comp. Surv. 23 (1991) 345-405.

[2] F.R. Chung, Spectral Graph Theory, American Mathematical Society, Providence, R. I., USA, CBMS 92 (1997).

[3] M.H. DeGroot and M.J. Schervish, Probability and Statistics, Pearson Addison Wesley, New York (2002).

[4] S. Herzel, M.C. Recchioni and F. Zirilli, A Quadratically Convergent Method for Linear Programming. Linear Algebra Appl. 152 (1991) 255-289.

[5] J.A. Snyman, Practical Mathematical Optimization: An Introduction to Basic Optimization Theory and Classical and New Gradient-Based Algorithms, Springer, Cambridge, Massachusetts, USA (2005). 